\title{
Galactic-scale star formation by gravitational instability
}

\section{Mordecai-Mark Mac Low ${ }^{1}$, Yuexing $\mathbf{L i}^{1,2}$ and Ralf S. Klessen ${ }^{3}$}

\author{
${ }^{1}$ Department of Astrophysics, American Museum of Natural History, 79th Street at Central \\ Park West, New York NY 10024-5192, USA. Email: mordecai@amnh.org \\ ${ }^{2}$ Harvard-Smithsonian Center for Astrophysics, Harvard University, 60 Garden Street, \\ Cambridge, MA 02138, USA. Email: yxli@cfa.harvard.edu \\ ${ }^{3}$ Institut für Theoretische Astrophysik, Zentrum für Astronomie der Universität Heidelberg, \\ Alber-Überle-Str. 2, D-69120 Heidelberg, Germany. Email: rklessen@ita.uni-heidelberg.de
}

\begin{abstract}
We present numerical experiments that demonstrate that the nonlinear development of the Toomre instability in disks of isothermal gas, stars, and dark matter reproduces the observed Schmidt Law for star formation. The rate of gas collapse depends exponentially on the (minimum) value of the Toomre parameter in the disk. We demonstrate that spurious fragmentation occurs in the absence of sufficient resolution in our SPH model. Our models also reproduce observed star formation thresholds in disk galaxies. We finally briefly discuss the application of our models to the study of globular cluster formation in merging galaxies.
\end{abstract}

Keywords. hydrodynamics, instabilities, galaxies: spiral

The tight correlation between gas surface density and star formation rate in galaxies demonstrated, for example, by Kennicutt (1998), suggests that the large-scale star formation rate seen in galaxies may be determined by relatively simple physics. Martin \& Kennicutt (2001) find thresholds in $\mathrm{H} \alpha$ emission that traces star formation, and suggest that these are caused by Toomre (1964) instability. (However, note the recent result from GALEX that the radial profiles of UV light emitted by young, massive stars in galaxies do not show the same breaks [e.g. Boissier et al. 2006].)

Star formation is a complex process where gravity and radiative cooling are opposed by thermal pressure, magnetic fields, rotation, and turbulent flows (e.g. Shu, Adams, \& Lizano 1987, Mac Low \& Klessen 2004). Yet, we are suggesting that relatively simple physics dominates the large-scale behavior. In the context of this conference, we are trying to raise the question of whether it is stellar triggering or gravitational instability that determines the large-scale star formation rate. Furthermore, we would like the same mechanism to explain how the very high star formation rates seen in starburst galaxies can be explained.

To address these questions we performed a series of numerical experiments, described in detail by Li, Mac Low, \& Klessen (2005b). We set up exponential disks of stars and gas embedded in a dark matter halo with a softened isothermal profile, and simulated their evolution using GADGET, a hybrid N-body/SPH code described by Springel, Yoshida, \& White (2001). In our experiments, we control the initial gravitational instability, and measure the properties of the collapsing gas, from which we infer the star formation rate and distribution. We follow regions of collapse using sink particles (Bate, Bonnell, \& Price 1995).

We made three major approximations, each of which has been studied in more detail with local computational models. First, we assumed that the gas behaved isothermally for purposes of computing its dynamics. Joung \& Mac Low (2006) demonstrated that 

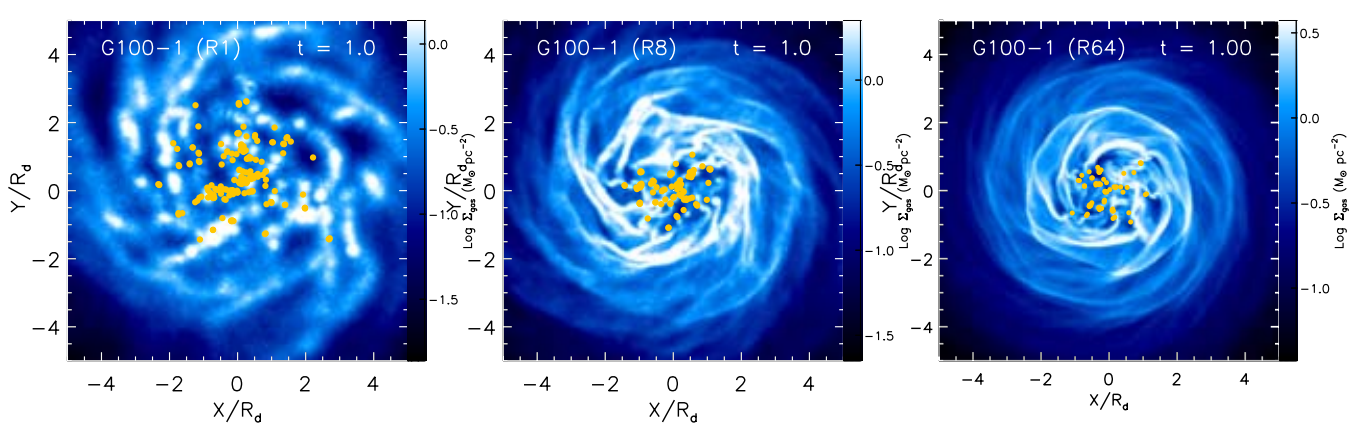

Figure 1. Gas surface density maps from a resolution study, showing models with total particle number of $N_{\text {tot }}=10^{5}(\mathrm{R} 1), 8 \times 10^{5}$ (R8) and $6.4 \times 10^{6}$ (R64). Model R1, which is underresolved according to the Jeans criterion of Bate \& Burkert (1997), shows strong spurious fragmentation. On the other hand model R8, which is marginally resolved, shows only minor differences with the highest-resolution model R64, suggesting the criterion is adequate. From Li, Mac Low, \& Klessen (2005b).

a supernova-driven ISM actually is rather more compressible than isothermal, so this is actually a conservative assumption. (Note though, that Robertson et al. (2004) and others have suggested that use of an isothermal equation of state does not produce stable galactic disks, a result we disagree with, as is discussed below.) Second, we assumed that molecular gas forms quickly once collapse sets in, which has been shown by Glover \& Mac Low (2006) using 3D non-equilibrium chemistry simulations to be consistent with the behavior of high-density, turbulent, magnetized, self-gravitating gas. Third, we neglected magnetic fields at these large scales, as they are unlikely to be dynamically important for gas collapsing after reaching the Jeans mass of $\sim 10^{6} \mathrm{M}_{\odot}$.

The major result that we draw from our experiments is that nonlinear development of gravitational instability quantitatively determines star formation properties.

Before we discuss the results in detail we need to discuss the three numerical criteria that models of gravitational collapse must satisfy. Most important is the Jeans resolution criterion (Bate \& Burkert 1997; Truelove et al. 1997; Whitworth 1998; Nelson 2006). Also necessary to be satisfied are the gravity-hydro balance criterion for gravitational softening (Bate \& Burkert 1997; Nelson 2006), and the equipartition criterion for particle masses (Steinmetz \& White 1997).

In Figure 1 we show a resolution study for one of our models, in which we increase the linear resolution by a factor of two between models (equivalent to increasing the mass resolution by a factor of 8 ). The lowest resolution model violates the Jeans criterion, while the two higher resolution models satisfy it. Spurious fragmentation can be clearly seen in the lowest resolution model, demonstrating that SPH simulations are indeed as subject as any other method to this problem, in contradiction to the claim of Hubber, Goodwin, \& Whitworth (2006). Robertson et al. (2004) and others have presented models of disk galaxies using an isothermal equation of state that collapsed because of the formation of large clumps that lost angular momentum due to dynamical friction, falling to the center of the disk and producing far too high a star formation rate. We believe this occurred due to spurious fragmentation rather than the limitation of the equation of state.

A linear analysis of axisymmetric gravitational instability was performed for collisionless stars by Toomre (1964), giving an instability parameter $Q_{s}=\kappa \sigma_{s} /\left(3.36 G \Sigma_{s}\right)$, and for collisional gas by Goldreich \& Lynden-Bell (1965), giving $Q_{g}=\kappa c_{g} /\left(\pi G \Sigma_{g}\right)$, where $\kappa$ is the epicyclic frequency, $\Sigma_{s, g}$ are star and gas surface densities, $\sigma_{s}$ is the radial stellar velocity dispersion, and $c_{g}$ is the isothermal gas sound speed. Rafikov (2001) analyzed the 


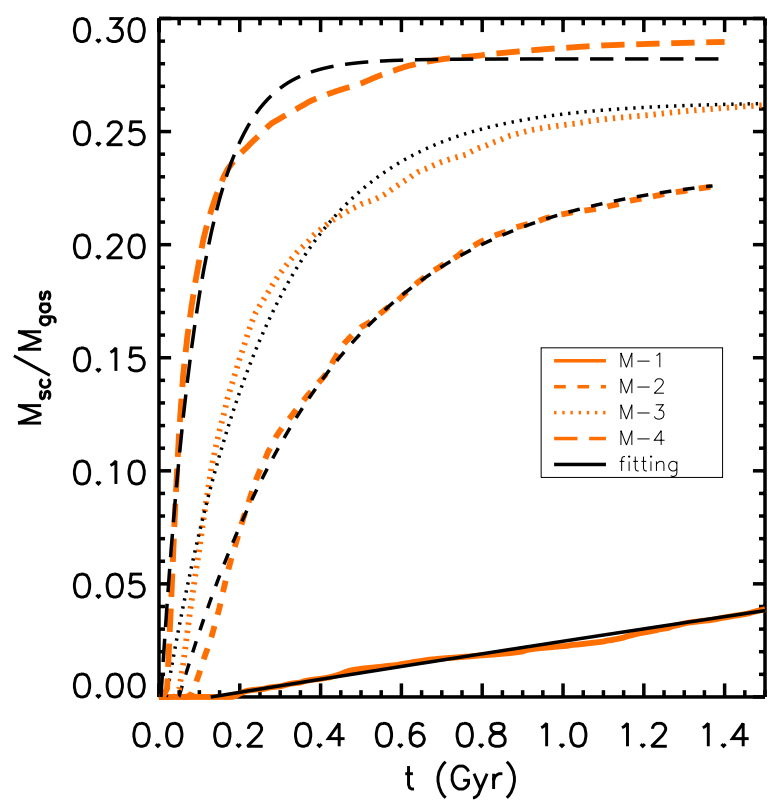

Figure 2. Time history of the mass in star clusters $M_{\mathrm{sc}}$ in several sample models, scaled by initial total gas mass $M_{\text {gas }}$, and compared to fits to equation (0.1). $M_{\mathrm{sc}}$ is taken as $30 \%$ of the mass of the sink particles. M-1 to 4 indicate submodels with increasing gas fractions. From Li, Mac Low, \& Klessen (2005b).

instability for a combination of collisionless stars and collisional gas, deriving a non-linear equation for a combined instability parameter $Q_{s g}$. We use this in our analysis.

The history of collapse in our models can be well fit by curves drawn from the single parameter family

$$
M_{s c}=M_{0}\left(1-\exp \left[-t / \tau_{\mathrm{sf}}\right]\right),
$$

as shown in Figure 2, where $M_{s c}$ is the total amount of collapsed mass, $M_{0}$ is the initial gas mass, and $\tau_{\text {sf }}$ is a collapse or star formation timescale. The collapse timescale $\tau_{\text {sf }}$ depends continuously in our model galaxies on the minimum initial value of $Q_{s g}$ in the disk, with an exponential dependence

$$
\tau_{\text {sf }} \propto \exp \left(\alpha Q_{s g}\right)
$$

where $\alpha \simeq 3$, as shown in Figure 3. This continuous dependence suggests that nominally stable galaxies can still show low rates of star formation, a conclusion that might extend to stable regions of galaxies as well. The detailed dependence of star formation on the local value of $Q_{s g}$ has been shown in preliminary results presented by Chu in this meeting. She showed a good correlation in the LMC between the location of young stellar objects detected in the Spitzer $8 \mu \mathrm{m}$ band and the local value of $Q_{s g}$, computed from the stellar, atomic, and molecular gas densities.

Combining equations (0.1) and (0.2), we can derive a nonlinear equation for the global star formation efficiency $\epsilon_{g}=M_{s c} / M_{0}=f\left(Q_{s g}\right)$. This predicts that the star formation efficiency in galaxies with $Q_{s g}>1$ drops continuously to less than $2 \%$ (see Li, Mac Low, \& Klessen 2006). 


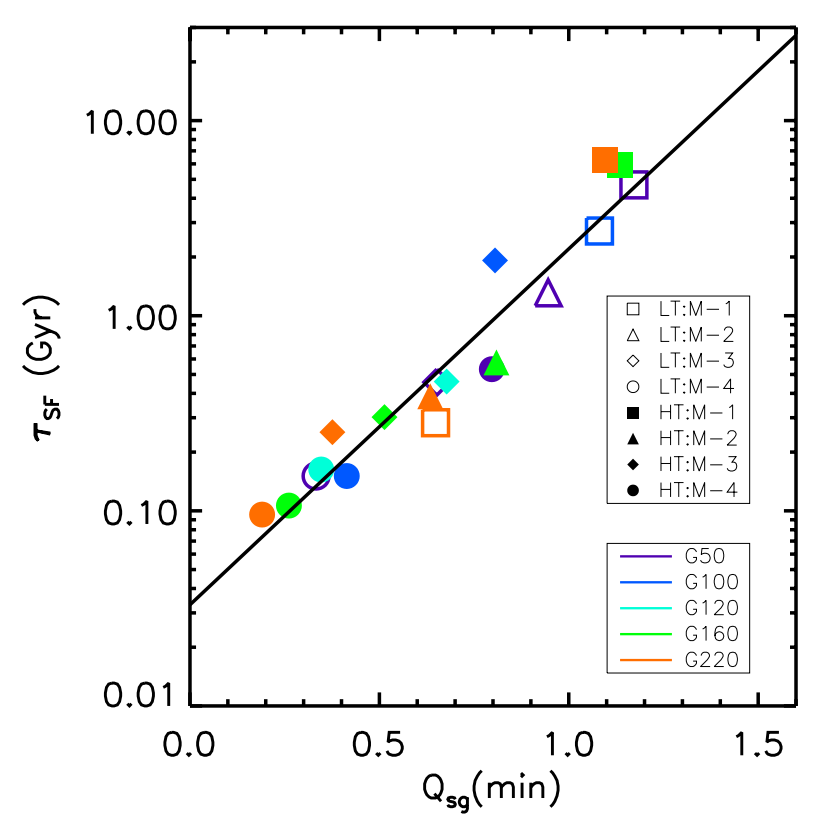

Figure 3. Star formation timescale $\tau_{\text {sf }}$ correlates with the initial disk instability $Q_{\mathrm{sg}}$ The solid line is a least-squares fits to the data. From Li, Mac Low, \& Klessen 2005b.

The total collapse rate in our galaxies can be measured at any time, allowing us to derive a quantity related to the star formation rate, and compare it to the gas surface density. As Figure 4 shows, our models fit the observed relation in both slope and normalization. In this plot, we used a constant conversion factor between collapse rate and star formation rate of $30 \%$ (a local efficiency for star formation in very unstable regions), but Li, Mac Low, \& Klessen (2006) demonstrate that local efficiencies within a reasonable range (i.e. $5-50 \%$ ) give similar results.

Other groups have recently derived the Schmidt Law in complementary ways. Kravtsov (2003) used an adaptive mesh refinement method to study gas collapse in an ensemble of galaxies evolving from cosmological initial conditions. Unlike many such simulations, he chose a star formation law $\dot{\rho}_{*} \propto \rho_{g}$, deliberately chosen to not reproduce the Schmidt law by design. Nevertheless, he found that the galaxies reproduced the Schmidt law, in agreement with our results. As he did not measure gravitational instability, he could not make the direct link that we do.

Krumholz \& McKee (2005), on the other hand, took the observed distributions of giant molecular clouds and H II regions as input. They assumed the clouds to be in virial equilibrium (an assumption studied more carefully by Krumholz, Matzner, \& McKee 2006), and then derived the Schmidt law from the properties of supersonic turbulence in the clouds. We argue that global gravitational instability determines the distribution of clouds and $\mathrm{H}$ II regions used in this approach.

Our models also reproduce the star formation thresholds found by Martin \& Kennicutt (2001). Li, Mac Low, \& Klessen (2005a) show that the radius within which $90 \%$ of star formation occurs is tied to the radius at which $Q_{s g}$ passes through unity. However, they also found that the more stable the galaxy, the larger the actual value of $Q_{s g}$ at threshold, over a range from 0.3 for extreme starbursts out to 1.5 for very stable galaxies. The 


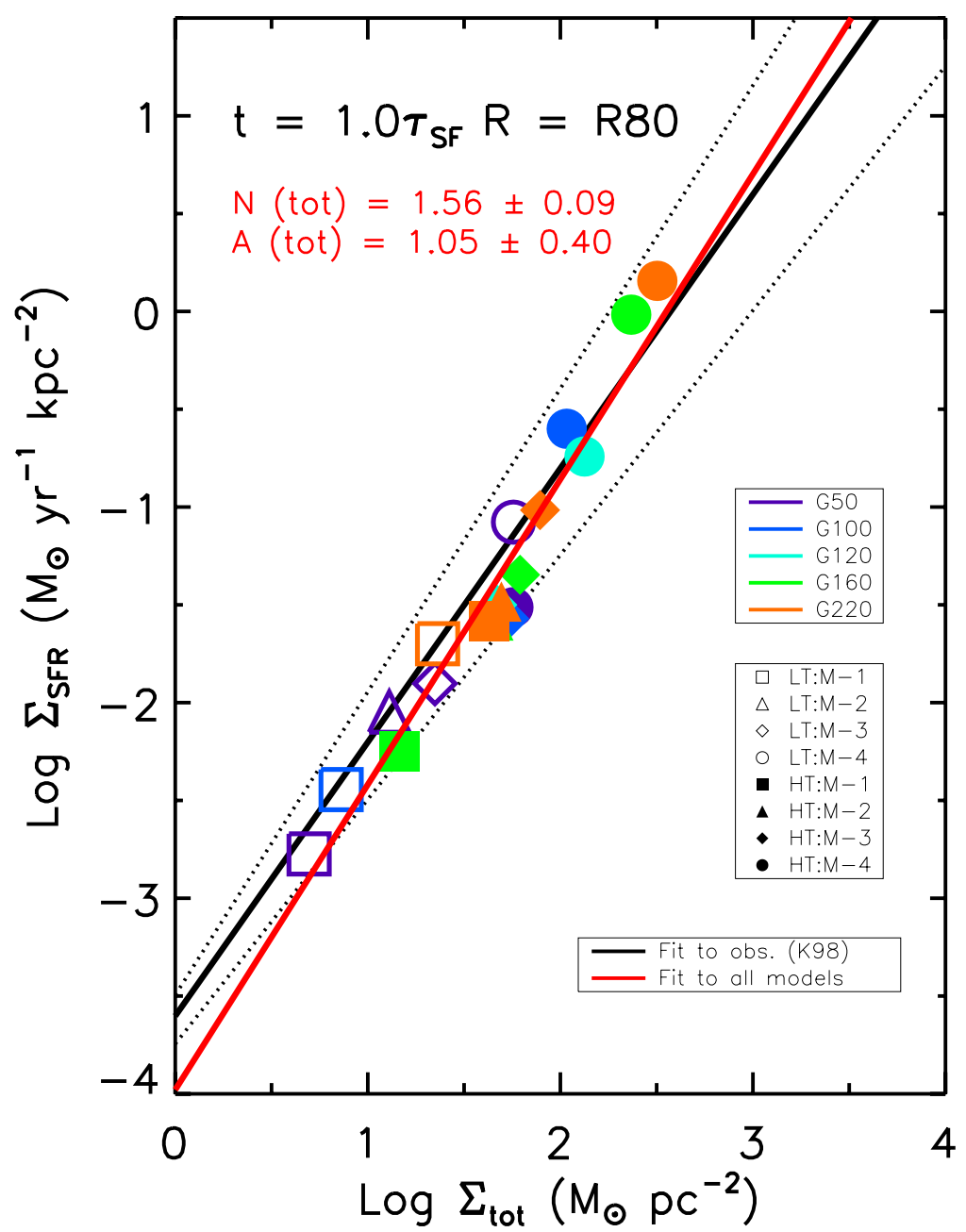

Figure 4. A comparison of the global Schmidt law between our simulations and the observations. The red line is the least-square fit to the total gas of the simulated models, the black solid line is the best fit of observations from Kennicutt (1998), while the black dotted lines indicate the observational uncertainty. The color of the symbol indicates the rotational velocity for each model, while labels from M-1 to M-4 are sub-models with increasing gas fraction; and open and filled symbols represent low and high temperature models, respectively. From Li, Mac Low, \& Klessen (2006).

observations of stable, modern galaxies by Martin \& Kennicutt (2001) have an average threshold value of $Q_{g}=1.4$, which appears consistent with our result.

We have used the same set of approximations to model globular cluster formation in merging galaxies, as described by Li, Mac Low, \& Klessen (2004). If we assume that each sink particle represents a cluster formation site, we find clear evidence for largescale triggering of star formation by galaxy mergers. Our results confirm the original suggestion by Ashman \& Zepf (1992) that globular clusters formed during galaxy mergers can explain the factor of three increase in observed specific frequency of globular clusters between spiral and elliptical galaxies.

We conclude by restating our major result: gravitational instability appears to be the dominant mechanism that controls the star formation rate in galaxies. Local triggering 
by OB stars appears to be roughly a 10-20\% effect (see talk by Mizuno in this meeting, and Joung \& Mac Low 2006). These conclusions agree with the basic principle laid out by Elmegreen (2002): gravitational instability determines the global star formation rate, but local triggering helps to determine exactly where and when star formation occurs. Finally, our results firmly support the idea that galaxy interactions represent an extremely effective form of global triggering.

\section{Acknowledgements}

We thank V. Springel for making both GADGET and his galaxy initial condition generator available, as well as for useful discussions, and A.-K. Jappsen for participating in the implementation of sink particles in GADGET. We are grateful to F. Adams, G. Bryan, J. Dalcanton, B. Elmegreen, D. Helfand, R. Kennicutt, J. Lee, C. Martin, R. McCray, T. Quinn, E. Quataert, M. Shara, C. Struck, and J. van Gorkom for very useful discussions. This work was supported by the NSF under grants AST99-85392 and AST03-07854, by NASA under grant NAG5-13028, and by the Emmy Noether Program of the DFG under grant KL1358/1. Computations were performed at the Pittsburgh Supercomputer Center supported by the NSF, on the Parallel Computing Facility of the AMNH, and on an Ultrasparc III cluster generously donated by Sun Microsystems.

\section{References}

Ashman, K. M. \& Zepf, S. E. 1992, ApJ 384, 50

Bate, M. R., Bonnell, I. A. \& Price, N. M. 1995, MNRAS 277, 362

Bate, M. R. \& Burkert, A. 1997, MNRAS 288, 1060

Boissier, S., et al. 2006, ApJ Suppl. in press (astro-ph/0609071)

Elmegreen, B. G. 2002, ApJ 577, 206

Goldreich, P. \& Lynden-Bell, D. 1965, MNRAS 130, 97

Glover, S. C. O. \& Mac Low, M.-M. 2006, ApJ submitted (astro-ph/0605121)

Hubber, D. A., Goodwin, S. P. \& Whitworth, A. P. 2006, A\&A 450, 881

Joung, M. K. R. \& Mac Low, M.-M. 2006, ApJ submitted (astro-ph/0601005)

Krumholz, M. R., Matzner, C. D. \& McKee, C. F. 2006, ApJ in press (astro-ph/0608471)

Krumholz, M. R. \& McKee, C. F. 2005, ApJ 630, 250

Kennicutt, R. C., Jr. 1998, ApJ 488, 541

Kravtsov, A. V. 2003, ApJ 590, L1

Li, Y., Mac Low, M.-M. \& Klessen R. S. 2004, ApJ 614, L29

Li, Y., Mac Low, M.-M. \& Klessen R. S. 2005a, ApJ 620, L19

Li, Y., Mac Low, M.-M. \& Klessen R. S. 2005b, ApJ 626, 823

Li, Y., Mac Low, M.-M. \& Klessen R. S. 2006, ApJ 639, 879

Mac Low, M.-M. \& Klessen, R. S. 2004, Rev. Mod. Phys. 76, 125

Martin, C. L. \& Kennicutt, R. C., Jr. 2001, ApJ 555, 301

Nelson, A. F. 2006, MNRAS in press (astro-ph/0609493)

Rafikov, R. R. 2001, MNRAS 323, 445

Robertson, B., Yoshida, N., Springel, V. \& Hernquist, L. 2004, ApJ 606, 32

Shu, F. H., Adams, F. C. \& Lizano, S. 1987, ARAA 25, 23

Springel, V., Yoshida, N. \& White S. D. M. 2001, New Astron. 6, 79

Steinmetz, M. \& White, S. D. M. 1997, MNRAS 288, 545

Toomre, A. 1964, ApJ 139, 1217

Truelove, J. K., Klein, R. I., McKee, C. F., Holliman, J. H., II, Howell, L. H. \& Greenough, J. A. $1997, A p J 489, \mathrm{~L} 179$

Whitworth, A. P. 1998, MNRAS 296, 442 


\section{Discussion}

BonNELL: Do the stars or the gas dominate the gravitational instabilities?

MAC Low: The large-scale gravitational potential is determined by the total mass, distributed among stars and gas. In model galaxies, the stars dominate the mass, though the cold gas can be locally important in regions where it is concentrated.

ELMEGREEN: You're suggesting Q can be much less than 1 and that starbursts and high efficiencies result from this, but in other global models, Q is self-regulated to stay near 1 and starbursts occur for the same reason as normal star formation, namely in marginally unstable gas with a great sensitivity to column density. Why do you prefer your model?

MAC Low: We find that low initial Q galaxies form stars with a very short timescale, so that the $\mathrm{Q}$ rises quickly towards unity. We need to compare star formation rates with the value of $\mathrm{Q}$ observable at that moment, rather than with the initial value. I hope to report on this soon.

TAN: I believe Downes \& Solomon (1998) measured $Q \sim 1$ in circumstellar starbursts, in contradiction with your results. I agree that gravitational instability controls where star formation occurs in galaxies (the threshold) but not that it sets the rate. This is because in most of the galaxies and starbursts defining the Kennicutt-Schmidt law, a large fraction $(\sim 0.5)$ of the gas is already in molecular clouds that are probably bound (McKee 1999), and that fraction is probably in an approximate steady state. The star formation rate is set by the rate at which these giant molecular clouds form star clusters, i.e., gravitational instabilities on scales much smaller than the galaxy, as in the models of Tan (2000) or Krumholz \& McKee (2005). I would thus argue that GMC formation from atomic gas is not the rate limiting step controlling galactic star formation rates.

MaC Low: I wonder if Downes \& Solomon (1998) included the stellar gravitational potential in their Q calculation. A factor of 2 is plenty, as the star formation rate is exponentially dependent on Q. As I understand the models of Tan (2000) or Krumholz \& McKee (2005), they depend on internal properties of the clouds (presumably universal) and on the observed distribution of molecular clouds in galaxies. We offer a theoretical grounding for that distribution, which appears to be the property that distinguishes galaxies with different star formation rates.

ZINNECKER: You said triggering would only change the global star formation efficiency by $10-10 \%$. Can I rephrase this by asking: If there were no supernovae whatsoever (just a thought experiment), would the star formation efficiency in galaxies be essentially the same?

MAC Low: Not necessarily. We assume an isothermal equation of state with an effective temperature of order $10^{4} \mathrm{~K}$. This implies strong energy input to maintain these temperatures or velocity dispersions. The SNe are a major candidate for this energy input, although as Eve Ostriker showed, there are many other candidates, particularly the magnetorotational instability. If the MRI provides a floor, then the SNe may not make a big difference.

DE Gouveia Dal Pino: The detailed calculation of the radiative coding and the implicit evolution of the chemical species have tremendous effects upon the structuring formation at the small scales (e.g., Melioli, de Gouveia Dal Pino, \& Raga, A\&A, 2005). Thus, to 
what extent does the adoption of an isothermal equation of state affect the time scales for global (large scale) structuring development and star formation in your simulations?

MAC LOW: I agree that the equation of state, determined by radiative cooling and nonequilibrium chemistry is vital to the details of fragmentation at small scales (e.g., Jappsen et al. 2005). However, our isothermal equation of state is primarily designed to simulate the dynamical effects of transonic or supersonic turbulence. The thermal state of the gas is less important at large scales.

CRUTCher: Without a detailed theory of star formation in simulations such as you present, it seems to me that what you get out may be dominated by your initial assumptions. For example, putting in global magnetic fields could profoundly affect the compression of clouds in the colliding galaxies.

MAC Low: The theory of gravoturbulent collapse does provide a reasonably well developed theory for what goes on inside sink particles, including magnetic effects (see Heitsch, Klessen, \& Mac Low 2001 and Glover \& Mac Low 2006). Whether global fields are important isn't directly addressed by our models yet. However, I suspect that the gravitational energy density far exceeds the magnetic energy density. 